\title{
A WEAK PROJECTION OF $C$ ONTO A EUCLIDEAN SUBSPACE
}

\author{
BY \\ EDWARD SILVERMAN $\left.{ }^{1}\right)$
}

In general there exists no projection of a space of continuous functions onto a subspace with the property that the distance between the projections of any two points does not exceed the distance between the two points. Nevertheless, we shall see that there is a projection of $C\left(S^{n-1}\right)$ onto a suitable Euclidean $n$-dim subspace with the property that the volume of the projection of a $k$-dim box does not exceed the volume of the box if $k>1$. The specific definitions are given later, but we can say here that volume, as defined in an $n$-dim Minkowski space, is equivalent to saying that the smallest box containing the unit sphere has volume $2^{n}$.

We then prove a weak Kirzbraun type theorem which enables us to deduce that Kolmogorov's Principle, as applied to Lebesgue area, holds for surfaces in $C$. From this we conclude that a generalization of Lebesgue area is an extension. Previously it was known that the generalization agreed with Lebesgue area only on those surfaces for which a lower area agreed with Lebesgue area.

The crucial result is, essentially, the following: Let

and

$$
f(x)=a_{0} / 2+\sum\left(a_{n} \cos n x+b_{n} \sin n x\right)
$$

Then

$$
g(x)=\alpha_{0} / 2+\sum\left(\alpha_{n} \cos n x+\beta_{n} \sin n x\right) .
$$

$$
\left|a_{1} \beta_{1}-b_{1} \alpha_{1}\right| \leqq \max _{x, y}[f(x) g(y)-f(y) g(x)] .
$$

We will frequently use the methods of $[S 1, \S 5]$. In fact, the idea of this paper, as in that, is to study a function by means of a suitably constructed convex function.

For the notation in Lemma 5, see [F].

1. Let $V$ be a normed vector space. If $b=\left(b^{1}, \ldots, b^{n}\right) \in V^{n}$ let $B$ be the vector subspace of $V$ spanned by $b$. Let $V$ be normed by $\mid$ and $V^{n}$ by $\|$ where $\|b\|^{2}=\sum\left|b^{i}\right|^{2}$.

If $K$ is a $n \times n$ matrix let $K^{*} \in L\left(V^{n}, V^{n}\right)$ be defined by $K^{*} b=c$ where $c^{i}=\sum_{j} K_{j}^{i} b^{j}$. We shall write $K b$ for $K^{*} b$. Let $K, T, U$ and $\Delta$ be $n \times n$ matrices of determinant one where, in addition, $T$ is triangular with positive entries on the diagonal, $U$ is orthogonal and $\Delta$ is diagonal with positive entries on the diagonal. Let $S$ be an arbitrary $n \times n$ matrix. We recall that Trace $S=\left.(d / d \varepsilon) \operatorname{det}[I+\varepsilon S]\right|_{\varepsilon=0}$. Thus Trace $T \geqq n$ and Trace $\Delta \geqq n$.

Received by the editors November 1\%, 1967.

( ${ }^{1}$ ) This research was supported in part by National Science Foundation Grant GP-04088. 
Let $\wedge b=b^{1} \wedge \cdots \wedge b^{n}$. If $\zeta=\left(\zeta_{1}, \ldots, \zeta_{n}\right)$, where each $\zeta_{i}$ is a continuous linear functional over $V$, then $\wedge \zeta=\zeta_{1} \wedge \cdots \wedge \zeta_{n}$ and $[\wedge b, \wedge \zeta]=\operatorname{det}\left[\zeta_{i}\left(b^{j}\right)\right]$.

If $P$ is a norm on $V^{n}$ then we say that $\zeta$ supports $P$ at $b$ if $\zeta(b)=P(b)$ and $\zeta \leqq P$. If $\zeta$ supports $P$ at $b$ and if $\zeta_{i}\left(b^{j}\right)=\delta_{i}^{j} P(b) / n$ for all $1 \leqq i, j \leqq n$, then $\zeta$ supports $P$ properly at $b$.

Lemma 1. If $\zeta$ supports $P$ properly at $b$ then $P(S b) \geqq \zeta(S b)=($ Trace $S) P(b) / n$.

The proof is immediate.

Let $Q$ and $M$ be norms on $V^{n}$ defined by $Q(b)=\|b\| \sqrt{ } n$ and $M(b)=\max _{U} Q(U b)$. Let $\check{Q}$ and $\hat{M}$ be norms on $\bigwedge^{n} V$ defined by $\mathscr{Q}(\bigwedge b)=n^{-n} \sup _{K} \inf _{T} Q^{n}(T K b)$ and $\hat{M}(\bigwedge b)=n^{-n} \inf _{K} M^{n}(K b)$. The norm, on $\bigwedge^{n} V$, is defined by

$$
|\bigwedge b|=\sup \left\{[\bigwedge b, \bigwedge \zeta]|| \zeta_{1}|=\cdots=| \zeta_{n} \mid=1\right\} .
$$

LEMMA 2. In the above definitions each supremum is a maximum, each infimum is a minimum and $\hat{M}(\bigwedge b)=n^{-n} \min _{T} M^{n}(T b)$.

Proof. Because of the Hahn-Banach Theorem, we can suppose that $V=B$. In addition, we can suppose that $\bigwedge b \neq 0$. Hence we can choose $\zeta$ so that $\zeta_{i}\left(b^{j}\right)=\delta_{i}^{j}$. Then $M(K b) \geqq Q(K b)=\|K b\| \sqrt{ } n \geqq\left|\sum_{j} K_{j}^{i} b^{j}\right| \sqrt{ } n$ for each $i$ so that $\|\zeta\| M(K b)$ $\geqq\left|\zeta_{p}\right|\left|\sum_{j} K_{j}^{i} b^{j}\right| \sqrt{ } n \geqq\left|\sum_{j} K_{j}^{i} \delta_{j}^{p}\right| \sqrt{ } n=\left|K_{p}^{i}\right| \sqrt{ } n$ for all $i$ and $p$. Thus in the definition of $\hat{M}, K$ may be restricted to lie in a compact set. A similar argument holds for $\mathscr{Q}$ and | Finally, for each $K$ there exists $U$ and $T$ such that $U K=T$.

Let $\hat{P}(\bigwedge b)=n^{-n} \min _{K} P^{n}(K b)$.

LEMMA 3. If $n^{n} P(\bigwedge b)=P^{n}(b)$ and if $\zeta_{i}\left(b^{j}\right)=\delta_{i}^{j} P(b) / n$, then $\zeta$ supports $P$ properly at $b$.

Proof. For each $S$ let $S_{m}=I+S / m$. Then det $S_{m}=1+($ Trace $S) / m+o\left(m^{-1}\right)$ as $m \rightarrow \infty$. Since $m S_{m}=m I+S, m P\left(S_{m} b\right) \leqq m P(b)+P(S b)$ so that

$$
P(S b)=m\left[P\left(S_{m} b\right)-P(b)\right] \geqq m\left[\left|\operatorname{det} S_{m}\right|^{1 / n}-1\right] P(b)
$$

since $P^{n}\left(S_{m} b\right) \geqq n^{n} \hat{P}\left(\bigwedge S_{m} b\right)=\left|\operatorname{det} S_{m}\right| P^{n}(b)$. Hence

$$
\begin{aligned}
P(S b) & \geqq m\left[(\text { Trace } S) /(m n)+o\left(m^{-1}\right)\right] P(b) \\
& =(\operatorname{Trace} S)[P(b)] / n+m o\left(m^{-1}\right) .
\end{aligned}
$$

Thus $\zeta(S b)=($ Trace $S) P(b) / n \leqq P(S b)$.

LEMMA 4. If $n^{n} \hat{P}(\bigwedge b)=P^{n}(b)$ then there exists $\zeta$ such that $\zeta$ supports $P$ properly at $b$ and $\hat{P}(\bigwedge b)=[\bigwedge b, \bigwedge \zeta]$.

Proof. The first part of the lemma is a consequence of the last lemma and the Hahn-Banach Theorem. Finally, $n^{n} \hat{P}(\bigwedge b)=P^{n}(b)=n^{n}[\bigwedge b, \bigwedge \zeta]$.

If $\zeta$ supports $P$ properly for some $b$, then we say that $\zeta$ supports $P$ properly.

LeMma 5. $\hat{M}(\bigwedge b)=\max \{[\bigwedge b, \bigwedge \zeta] \mid \zeta$ supports $N$ properly $\}$. 
Proof. Let $\eta$ support $M$ properly. Because of Lemma 4 we need only show that $[\bigwedge b, \bigwedge \eta] \leqq \hat{M}(\bigwedge b)$ so that we may assume that $[\wedge b, \wedge \eta]>0$. We can also assume that $\hat{M}(\bigwedge b)=n^{-n} M^{n}(b)$. Let $\eta_{i}^{j}=\eta_{i}\left(b^{j}\right), \quad v_{n}=*\left[\eta_{1} \wedge \cdots \wedge \eta_{n-1}\right], v_{n-1}$ $=*\left[\eta_{1} \wedge \cdots \wedge \eta_{n-2} \wedge v_{n}\right], \ldots, v_{1}=*\left[v_{2} \wedge v_{3} \wedge \cdots \wedge v_{n}\right]$. Thus $v_{i} \cdot \eta_{j}=0$ for $i>j$ and $v_{i} \cdot \eta_{i} \neq 0$ for all $i$. By replacing $v_{i}$ with $\pm v_{i}\left|v_{i}\right|^{-1}$ we can suppose that $\left\{v_{i}\right\}$ is an orthonormal set, $v_{i} \cdot \eta_{j}=0$ for $i>j$ and $v_{i} \cdot \eta_{i}>0$. Now let $u_{i}^{j}=v_{j}^{i}$. Then $U=\left[u_{i}^{j}\right]$ is an orthogonal matrix and $c^{j}=(U b)^{j}=\sum u_{k}^{j} b^{k}=\sum v_{j}^{k} b^{k}$ so that $\eta_{i}\left(c^{j}\right)=v_{j} \cdot \eta_{i}$. Now we have $[\bigwedge b, \bigwedge \eta]=[\bigwedge c, \bigwedge \eta]=\prod_{i=1}^{n} \eta_{i}\left(c^{i}\right) \leqq\left\{n^{-1} \sum \eta_{i}\left(c^{i}\right)\right\}^{n}=n^{-n} M^{n}(c)=n^{-n} M^{n}(b)$ $=M(\bigwedge b)$.

LEMMA 6. $\check{Q}=\hat{M}$.

Proof. We can suppose that $\bigwedge b \neq 0$ and that $V=B$. Since $n^{n} \hat{M}(\bigwedge b)$ $=n^{n} \hat{M}(\bigwedge K b)=\min _{T} M^{n}(T K b) \geqq \min _{T} Q^{n}(T K b)$ for all $K, \hat{M}(\bigwedge b) \geqq \grave{Q}(\bigwedge b)$. On the other hand, there exist $U_{0}$ and $T_{0}$ such that $n^{n} \hat{M}\left(\bigwedge U_{0} T_{0} b\right)=n^{n} \hat{M}(\bigwedge b)$ $=M^{n}\left(T_{0} b\right)=Q^{n}\left(U_{0} T_{0} b\right)=M^{n}\left(U_{0} T_{0} b\right)$. Let $d=U_{0} T_{0} b$. Suppose that $Q^{\prime}(d)$ and $M^{\prime}(d)$ both exist. Since $Q$ and $M$ are convex, $M \geqq Q$ and $M(d)=Q(d)$, it follows that $Q^{\prime}(d)=M^{\prime}(d)$. Let $\zeta=M^{\prime}(d)$. By Lemma 4, $\zeta_{i}\left(d^{j}\right)=\delta_{i}^{j} M(d) / n=\delta_{i}^{j} Q(d) / n$ so that, by Lemma $1, Q(T d) \geqq Q(d)=M(d)$ for all $T$. Hence $\mathscr{Q}(\bigwedge b)=\check{Q}(\bigwedge d)$ $\geqq \hat{M}(\bigwedge d)=\hat{M}(\bigwedge b)$. If $Q^{\prime}(d)$ or $M^{\prime}(d)$ does not exist, let $Q_{m}$ be a sequence of smooth uniformly convex norms approaching $Q$ from below. As before there exist $U_{m}$ and $T_{m}$ such that $Q_{m}\left(d_{m}\right)=M_{m}\left(d_{m}\right)$ where $d_{m}=U_{m} T_{m} b$. If there is a unique $U_{m}$ satisfying the required condition, then $M_{m}$ is also differentiable in a neighborhood of $d_{m}$ by the implicit function theorem. Otherwise, we can modify $Q_{m}$ slightly to obtain the desired condition. Thus $\check{Q}=\lim \check{Q}_{m}=\lim \hat{M}_{m}=\hat{M}$.

We have proved

Lemma 7. Let $b \in V^{n}$. Then there exists $d \in V^{n}$ with $\bigwedge d=\bigwedge b$ and $n^{n} \hat{M}(\bigwedge d)$ $=M^{n}(d)=Q^{n}(d)$.

LEMMA 8. If $n^{n} \hat{M}(\bigwedge d)=M^{n}(d)=Q^{n}(d)$ then $N\left(d^{i}, d^{j}\right)=\eta\left(d^{i}, d^{j}\right)$ for all $1 \leqq i, j \leqq n$ where $2 \eta\left(d^{i}, d^{j}\right)=\left|d^{i}\right|^{2}+\left|d^{j}\right|^{2}$ and

$$
N\left(d^{i}, d^{j}\right)=\operatorname{Max}_{\theta} \eta\left(d^{i} \cos \theta-d^{j} \sin \theta, d^{i} \sin \theta+d^{j} \cos \theta\right) .
$$

Proof. Let $U$ be the $n \times n$ matrix whose upper left corner is

$$
\left[\begin{array}{rr}
\cos \theta & -\sin \theta \\
\sin \theta & \cos \theta
\end{array}\right]
$$

and whose lower right corner is the $(n-2) \times(n-2)$ identity matrix. If $U d=e$ then, by the hypothesis, $Q(d) \geqq Q(e)$. Thus

$$
\sum\left|d^{i}\right|^{2} \geqq\left|e^{i}\right|^{2}=\left|d^{1} \cos \theta-d^{2} \sin \theta\right|^{2}+\left|d^{1} \sin \theta+d^{2} \cos \theta\right|^{2}+\sum_{i>2}\left|d^{i}\right|^{2}
$$

so that $\eta\left(d^{1}, d^{2}\right)=N\left(d^{1}, d^{2}\right)$. A similar argument holds for all $i<j$. 
Let $c^{1}, \ldots, c^{n} \in B$. If $c=S b$ and if $|\operatorname{det} S| \leqq 1$ then we say that the volume of the box spanned by $c$ does not exceed the volume of the box spanned by $b$, even though we have not defined volume in $B$. We observed in [S3] that the definition of $|\wedge b|$ is equivalent to setting equal to $2^{n}$ the volume of the smallest box in $B$ which contains the unit sphere. If the sides of such a box $P$ are $\eta_{i}= \pm 1$, where $\left|\eta_{i}\right|=1, i=1, \ldots, n$, then there exist $c^{i}$ with $\left|c^{i}\right|=1$ and $\eta_{i}\left(c^{i}\right)=1$. Suppose $\eta_{j}\left(c^{i}\right) \neq 0$ for some $j \neq i$. Then let $d^{i}$ be determined by the conditions $\eta_{j}\left(d^{i}\right)=0$ for $j \neq i,\left|d^{i}\right|=1$ and $\eta_{i}\left(d^{i}\right)>0$. Now set $\xi_{j}=\eta_{j}$ for $j \neq i$ and let $\xi_{i}$ have norm one and support the unit sphere at $d^{i}$. It is clear from the geometry that the box whose sides are $\xi_{k}= \pm 1$ has volume not exceeding that of $P$. Hence we can suppose that $\eta_{i}\left(c^{j}\right)=\delta_{i}^{j}$. Furthermore, by the Cauchy inequality, if $d \in V^{n}$ then $\eta(d)=\sum \eta_{i}\left(d^{i}\right) \leqq \sum\left|d^{i}\right| \leqq Q(d)$ so that $\eta$ supports $Q$ properly at $c$.

THEOREM 1. $\hat{M}(\bigwedge b)=|\bigwedge b|=\check{Q}(\bigwedge b)$ for all $\bigwedge b \in \bigwedge^{n} V$.

Proof. We can suppose that $\bigwedge b \neq 0$. By the preceding remarks, we can assume that $\eta$ supports $Q$ properly at $b$ and that $|\wedge b|=[\wedge b, \wedge \eta]$. By Lemma $1, Q(T K b)$ $\geqq Q(b)$ for all $T K$ so that $\mathscr{Q}(\bigwedge b) \geqq n^{-n} Q^{n}(b)=[\wedge b, \bigwedge \eta]=|\wedge b|$. On the other hand, if $\zeta$ supports $Q$ properly at $d$, with $\wedge d \neq 0$, and if $f(\Delta)=Q(\Delta d)$, then $f$ has a minimum at $I$ so that $\left|d^{i}\right|=Q(d) / n$ for each $i$ : if $\left|d^{1}\right|=k^{2}\left|d^{2}\right|$ with $k \neq \pm 1$, then $\left.\left|d^{1}\right| k\right|^{2}+\left|k d^{2}\right|^{2} \leqq\left|d^{1}\right|^{2}+\left|d^{2}\right|^{2}$. Now let $e \in V$. Then

$$
\begin{aligned}
Q(d)\left[\zeta_{1}(e)+(n-1)|e|\right] / n & =\zeta\left(\left|d^{1}\right| e,|e| d^{2}, \ldots,|e| d^{n}\right) \\
& \leqq Q\left(\left|d^{1}\right| e,|e| d^{2}, \ldots,|e| d^{n}\right)=Q(|e| d)=|e| Q(d) .
\end{aligned}
$$

Hence $\zeta_{1}(e) \leqq|e|$ so that, by replacing $e$ with $-e$, we see that $\left|\zeta_{1}\right| \leqq 1$. It follows that $\left|\zeta_{1}\right|=1$ and, similarly, $\left|\zeta_{i}\right|=1$ for all $i$. Hence $\mathscr{Q}(\bigwedge d)=n^{-n} Q^{n}(d)=[\bigwedge d, \bigwedge \zeta]$ $\leqq|\wedge d|$ and an application of Lemma 6 completes the proof.

Lemma 9. Let $X$ be a compact Hausdorff space and $V=C(X)$. If

$$
f=\left(f^{1}, \ldots, f^{n}\right) \in V^{n}
$$

then $|\bigwedge f|=R$ where $R=\max \left\{\operatorname{det}\left[f^{i}\left(x_{j}\right)\right] \mid x_{1}, \ldots, x_{n} \in X\right\}$.

Proof. Let $y_{1}, \ldots, y_{n} \in X$ and $\mu_{j}$ be the measure on $X$ which has unit mass at $y_{j}$. Let $\zeta_{j}(f)=f\left(y_{j}\right)$. Then $\operatorname{det}\left[f^{i}\left(y_{j}\right)\right]=\operatorname{det}\left[\zeta_{j}\left(f^{i}\right)\right]=[\wedge \zeta, \wedge f] \leqq|\wedge f|$. On the other hand if $\zeta_{1}, \ldots, \zeta_{n}$ are linear functionals of norm one over $C(X)$ there exist measures $\lambda_{i}$ with $\int\left|d \lambda_{i}\right|=1$ such that $\zeta_{i}(f)=\int f d \lambda_{i}$ for all $f \in C(X)$. Hence

$$
[\bigwedge \zeta, \wedge f]=\operatorname{det}\left[\zeta_{i}\left(f^{j}\right)\right]=\int_{X} \cdots \int_{X} \operatorname{det}\left[f^{j}\left(x_{i}\right)\right] d \lambda_{1}\left(x_{1}\right) \cdots d \lambda_{n}\left(x_{n}\right) \leqq R .
$$

An interesting consequence is the following: if $f \in V^{n}$ there exists $K$ and $x_{1}, \ldots, x_{n} \in X$ such that, if we set $g^{i}=(K f)^{i}$,

$$
\left|g^{i}\right|=c \text { and } g^{i}\left(x_{j}\right)=\delta_{j}^{i} c
$$

where $c^{n}=|\bigwedge f|$ 
2. Let $A^{1}=[-\pi, \pi], A^{k+1}=A^{k} \times[0, \pi]$ and $S^{m}$ be the unit $m$-sphere canonically embedded in $R^{m+1}$ for some $m \geqq 1$. Let $\tau: A^{m} \rightarrow R^{m+1}$ be defined by

$$
\begin{aligned}
\tau^{i}(a) & =\cos a^{i} \sin a^{i+1} \cdots \sin a^{m}, \quad 1 \leqq i<m, \\
\tau^{m}(a) & =\cos a^{m} \text { and } \\
\tau_{m}^{m+1}(a) & =\sin a^{1} \sin a^{2} \cdots \sin a^{m} .
\end{aligned}
$$

Let $\mu_{m}(a)=\sin a^{2} \sin ^{2} a^{3} \cdots \sin ^{m-1} a^{m}$ so that $\int_{A^{m}} \mu_{m}(a) d a=K_{m}=$ area $S^{m}$.

If $f, g \in C\left(S^{m}\right)$ let

$$
(f, g)=\int_{S^{m}} f g d \lambda_{m}=K_{m}^{-1} \int_{A^{m}}\left(f \circ \tau_{m}\right)\left(g \circ \tau_{m}\right) \mu_{m}
$$

where $\lambda_{m}$ is Haar measure on $S^{m}$. If $x \in S^{m}$ let $\sigma^{0}(x)=K_{m}^{-1 / 2}$ and $\sigma^{i}(x)=K_{m}^{-1 / 2}(m+1)^{1 / 2} x^{i}$ for $i=1,2, \ldots, m+1$. Then $\left(\sigma^{i}, \sigma^{j}\right)=\delta_{j}^{i}$. Let $E_{m+1}$ be the vector subspace of $C\left(S^{m}\right)$ spanned by $\sigma^{1}, \ldots, \sigma^{m+1}$ and let $P, Q: C\left(S^{m}\right) \rightarrow E_{m+1}$ be defined by

$$
P f=\sum_{i=1}^{m+1}\left(f, \sigma^{i}\right) \sigma^{i}
$$

and

$$
Q f=\left(f, \sigma^{1}\right) \sigma^{1}+\left(f, \sigma^{2}\right) \sigma^{2} .
$$

If $f=\sum_{i=1}^{m+1} f_{i} \sigma^{i}$ then $|f|=K_{m}^{-1 / 2}(m+1)^{1 / 2}\left[\sum f_{i}^{2}\right]^{1 / 2}$ so that $E_{m+1}$ is a Euclidean subspace of $C\left(S^{m}\right)$.

Let $\eta(f, g)=\left[|f|^{2}+|g|^{2}\right] / 2$ and

$$
N(f, g)=\max _{\theta} \eta(f \cos \theta-g \sin \theta, f \sin \theta+g \cos \theta) .
$$

If $f, g \in E_{m+1}$ then $N(f, g)=\eta(f, g)$.

Lemma 10. Let $\gamma_{m}=\prod_{j=2}^{m}\left\{\int_{0}^{\pi} \sin ^{j} a^{j} d a^{j}\right\}$. Then $\gamma_{m}=K_{m+1} /(4 \pi)$.

Proof. $K_{m+1}=\int_{-\pi}^{\pi}\left\{\int_{0}^{\pi} \gamma_{m} \sin a^{1} d a^{1}\right\} d a^{0}=4 \pi \gamma_{m}$.

LEMMA 11. $N(Q f, Q g) \leqq N(f, g)$.

Proof. If there exist $F$ and $G$ with $N(Q f, Q G) \geqq N(Q f, Q g)$ and $N(F, G) \leqq N(f, g)$, then it is sufficient to prove the lemma with $f$ and $g$ replaced by $F$ and $G$, respectively.

If $h \in C\left(S^{m}\right)$ let

$$
\tilde{h} \circ \tau(a)=\frac{4 \pi}{K_{m+1}} \int_{0}^{\pi} \cdots \int_{0}^{\pi} h(\tau(a))\left\{\prod_{j=2}^{m} \sin ^{j} a^{j}\right\} d a^{2} \cdots d a^{m} .
$$

Then $\left(\tilde{h}, \sigma^{1}\right)=\left(h, \sigma^{1}\right)$ and $\left(\tilde{h}, \sigma^{m+1}\right)=\left(h, \sigma^{m+1}\right)$ so that $Q \tilde{h}=Q h$. In addition, $\tilde{h} \circ \tau$ is independent of $a^{2}, \ldots, a^{m}$. Let $T: E_{m+1} \rightarrow E_{2}$ be defined by $T\left(h \circ \tau_{m}\right)(a)=h \circ$ $\tau_{m}\left(a^{1}, \pi / 2, \ldots, \pi / 2\right)$. Then $T\left(\sigma^{1} \circ \tau\right)(a)=\cos a^{1}, T\left(\sigma^{m+1} \circ \tau\right)(a)=\sin a^{1}$ and $T$ restricted to the plane determined by $\sigma^{1} \circ \tau$ and $\sigma^{m+1} \circ \tau$ is an isometry. Hence 
$N(T Q \tilde{f}, T Q \tilde{g})=N(Q \tilde{f}, Q \tilde{g})$. That $N(T \tilde{f}, T \tilde{g}) \leqq N(\tilde{f}, \tilde{g})$ is evident. Thus we can suppose that $m=1$. Furthermore, if we use the Weierstrass approximation theorem, there is no loss in generality in supposing that

$$
f \circ \tau_{1}(\theta)=\frac{a_{0}}{2}+\sum_{n=1}^{N}\left(a_{n} \cos n \theta+b_{n} \sin n \theta\right),
$$

and

$$
g \circ \tau_{1}(\theta)=\frac{\alpha_{0}}{2}+\sum_{n=1}^{N}\left(\alpha_{n} \cos n \theta+\beta_{n} \sin n \theta\right) .
$$

Let us now write $f$ for $f \circ \tau_{1}$ and $g$ for $g \circ \tau_{1}$. Thus $f$ and $g$ are defined on $[-\pi, \pi]$, but we shall not distinguish between these functions and their periodic of period $2 \pi$ extensions over all of $R^{1}$. Let $c_{k}$ and $s_{m}$ be defined by $c_{k}(\theta)=\cos k \theta$ and $s_{m}(\theta)$ $=\sin m \theta, k=0,1,2, \ldots$, and $m=1,2,3, \ldots$ As we have already noted, we can suppose that

$$
f=a_{0} / 2+\sum\left(a_{n} c_{n}+b_{n} s_{n}\right) \text { and } g=\alpha_{0} / 2+\sum\left(a_{n} c_{n}+\beta_{n} s_{n}\right)
$$

where the sums are finite sums. The change from $C\left(S^{1}\right)$ to the space of continuous, periodic functions on $[-\pi, \pi]$ is, of course, an isometry, and the lemma can be reformulated as follows: Let

$$
(f, g)=\int_{-\pi}^{\pi} f(\theta) g(\theta) d \theta \text { and } Q h=\frac{(h, c)}{(c, c)} c+\frac{(h, s)}{(s, s)} s .
$$

Then $\left|a_{1} \beta_{1}-b_{1} \alpha_{1}\right|=|Q f \wedge \dot{Q} g| \leqq N(f, g)$. Hence we can suppose that $a_{1}^{2}+\alpha_{1}^{2}>0$.

Let $F(\theta)=[f(\theta)-f(\pi+\theta)] / 2$ and $G(\theta)=[g(\theta)-g(\pi+\theta)] / 2$. Since $N$ is convex, $N(F, G) \leqq N(f, g)$ while $Q F=Q f$ and $Q G=Q g$. Hence we can suppose, in (1), that $a_{k}=b_{k}=\alpha_{k}=\beta_{k}=0$ if $k$ is not odd.

Let $p$ be chosen so that

$$
\left(a_{1} b_{1}+\alpha_{1} \beta_{1}\right) \cos 2 p+\left(a_{1}^{2}-b_{1}^{2}+\alpha_{1}^{2}-\beta_{1}^{2}\right) \sin 2 p=0 .
$$

Let $F(\theta)=f(\theta-p)$ and $G(\theta)=g(\theta-p)$. Then $N(F, G)=N(f, g)$ while

$$
Q F=\left(a_{1} \cos p-b_{1} \sin p\right) c_{1}+\left(a_{1} \sin p+b_{1} \cos p\right) s_{1}
$$

and

$$
Q G=\left(\alpha_{1} \cos p-\beta_{1} \sin p\right) c_{1}+\left(\alpha_{1} \sin p+\beta_{1} \cos p\right) s_{1} .
$$

Let $\Pi_{2}$ be the plane determined by $c_{1}$ and $s_{1}$. The induced metric on $\Pi_{2}$ is Euclidean so that $\eta=N$ on $\Pi_{2}$. Hence $N(Q F, Q G)=\eta(Q F, Q G)=\eta(Q f, Q g)=N(Q f, Q g)$. Hence, in (1), we can suppose that $a_{1} b_{1}+\alpha_{1} \beta_{1}=0$.

Now let $\cos w=a_{1} / r$ and $\sin w=-\alpha_{1} / r$ where $r=\left(a_{1}^{2}+\alpha_{1}^{2}\right)^{1 / 2}>0$. Then $\alpha_{1} \cos w$ $+a_{1} \sin w=b_{1} \cos w-\beta_{1} \sin w=0$. Let $F=f \cos w-g \sin w$ and $G=f \sin w+$ $g \cos w$. Then $N(F, G)=N(f, g)$ and $N(Q F, Q G)=N(Q f, Q g)$ so that we can suppose that $b_{1}=\alpha_{1}=0$. 
Let $\tilde{\psi}(\theta)=\psi(\pi-\theta), F=(f-\tilde{f}) / 2$ and $G=(g+\tilde{g}) / 2$. It is easy to see that $Q F=Q f$ and $Q G=Q g$. Since

$|F \cos u-G \sin u| \leqq[|f \cos u-g \sin u|+|\tilde{f} \cos u+\tilde{g} \sin u|] / 2$,

$|F \cos u-G \sin u|^{2} \leqq\left[|f \cos u-g \sin u|^{2}+|f \cos u+g \sin u|^{2}\right] / 2$

and similarly,

$$
|F \sin u+G \cos u|^{2} \leqq\left[|f \sin u+g \cos u|^{2}+|f \sin u-g \cos u|^{2}\right] / 2
$$

for all $u$ so that $N(F, G) \leqq N(f, g)$. Hence we can suppose that

$$
f=\sum a_{k} c_{k} \text { and } g=\sum \beta_{k} s_{k}
$$

where the summation is over a finite collection of odd natural numbers. Furthermore, we can suppose that $a_{1} \geqq 0$ and $\beta_{1} \geqq 0$.

Now let $\psi^{*}(\theta)=\psi(\theta+\pi 2), F=\left(f+g^{*}\right) / 2$ and $G=\left(g-f^{*}\right) / 2$. First we observe that $2 F^{*}=f^{*}-g=-2 G$ and, similarly, $G^{*}=F$, so that

$$
\begin{aligned}
N(F, G) & \geqq|F \wedge G| \geqq \max _{\theta}\left[F(\theta) G^{*}(\theta)-F^{*}(\theta) G(\theta)\right]=\left|F^{2}+G^{2}\right| \\
& \geqq \frac{1}{2 \pi} \int_{-\pi}^{\pi}\left[F^{2}(\theta)+G^{2}(\theta)\right] d \theta .
\end{aligned}
$$

Next we see that $|F \cos u-G \sin u| \leqq\left[|f \cos u-g \sin u|+\left|g^{*} \cos u+f^{*} \sin u\right|\right] / 2$ so that $|F \cos u-G \sin u|^{2} \leqq\left[|f \cos u-g \sin u|^{2}+|f \sin u+g \cos u|^{2}\right] / 2 \leqq N(f, g)$. Similarly, $|F \sin u+G \sin u|^{2} \leqq N(f, g)$ so that $N(F, G) \leqq N(f, g)$. An easy computation yields

$$
F=\sum_{k \in I} A_{k} c_{k}+\sum_{k \in J} A_{k} c_{k}
$$

and

$$
G=\sum_{k \in I} A_{k} s_{k}-\sum_{k \in J} A_{k} s_{k}
$$

where $I$ and $J$ are finite sets of odd natural numbers which are congruent to one and three, respectively, $\bmod 4$, and

Hence

$$
\begin{aligned}
A_{k} & =\left(a_{k}+\beta_{k}\right) / 2 \quad \text { if } k \in I, \\
& =\left(a_{k}-\beta_{k}\right) / 2 \quad \text { if } k \in J .
\end{aligned}
$$

$$
|Q F \wedge Q G|=A_{1}^{2} \leqq \sum A_{k}^{2}=\frac{1}{2 \pi} \int_{-\pi}^{\pi}\left[F^{2}(\theta)+G^{2}(\theta)\right] d \theta \leqq N(F, G) .
$$

THEOREM 2. If $f, g \in C\left(S^{m}\right)$ then $|P f \wedge P g| \leqq N(f, g)$.

Proof. If $U$ is an orthogonal $(n+1) \times(n+1)$ matrix and if $\phi \in C\left(S^{m}\right)$ let $U^{*} \phi$ $=\phi U^{-1}$. There exists $U$ such that $P\left(U^{*} f\right)=Q\left(U^{*} f\right)$ and $P\left(U^{*} g\right)=Q\left(U^{*} g\right)$. Thus $N(f, g)=N\left(U^{*} f, U^{*} g\right) \geqq\left|Q U^{*} f \wedge Q U^{*} g\right|=\left|P U^{*} f \wedge P U^{*} g\right|=|P f \wedge P g|$. 
TheOREM 3. If $f, g \in C\left(S^{m}\right)$ then $|P f \wedge P g| \leqq|f \wedge g|$.

Proof. There exist $F$ and $G$ such that $F \wedge G=f \wedge g$ and $N(F, G)=|F \wedge G|$. Hence $|P f \wedge P g|=|P F \wedge P G| \leqq N(F, G)=|F \wedge G|=|f \wedge g|$.

LEMmA 12. Let $a_{i}=\left(a_{i}^{1}, \ldots, a_{i}^{n}\right) \in R^{n}$ and $\Delta=\left|\operatorname{det}\left[a_{i}^{j}\right]\right|$. Then

$$
\Delta \leqq n^{-n}\left\{\frac{2 n}{n-1} \sum_{i<j}\left|a_{i} \wedge a_{j}\right|\right\}^{n / 2}
$$

Proof. There exists a rotation $U$ such that $[U a]$ is triangular. Hence we can suppose that $a_{i}^{j}=0$ for $j>i$. We can also suppose that $b_{i}=a_{i}^{i}>0$ for all $i$. In this case

$$
\Delta=\prod_{i=1}^{n} b_{i} \leqq\left\{\frac{2}{n(n-1)} \sum_{i<j} b_{i} b,\right\}^{n / 2}
$$

by a well-known arithmetic-geometric inequality. Since $\left|a_{i} \wedge a_{j}\right| \geqq b_{i} b_{j}$, the lemma follows.

THEOREM 4. Let $f^{1}, \ldots, f^{n} \in C\left(S^{n-1}\right)$. Then $|\bigwedge P f| \leqq|\bigwedge f|$.

Proof. We can suppose that $n^{n} \hat{M}(\bigwedge f)=M^{n}(f)=Q^{n}(f)$ by Lemma 7. Hence, by Lemma 8, Theorem 2 and Lemma 12,

$$
\begin{aligned}
|\wedge P f| & \leqq n^{-n}\left\{\frac{2 n}{n-1} \sum_{i<j}\left|P f^{i} \wedge P f^{j}\right|\right\}^{n / 2} \\
& \leqq n^{-n}\left\{\frac{2 n}{n-1} \sum_{i<j} N\left(P f^{i}, P f^{j}\right)\right\}^{n / 2} \\
& \leqq n^{-n}\left\{\frac{2 n}{n-1} \sum_{i<j} N\left(f^{i}, f^{j}\right)\right\}^{n / 2} \\
& =n^{-n}\left\{\frac{n}{n-1} \sum_{i<j}\left(\left|f^{i}\right|^{2}+\left|f^{j}\right|^{2}\right)\right\}^{n / 2} \\
& =n^{-n}\left\{n \sum_{i=1}^{n}\left|f^{i}\right|^{2}\right\}^{n / 2}=n^{-n} Q^{n}(f)=\hat{M}(\bigwedge f)=|\wedge f| .
\end{aligned}
$$

3. We will recall the definitions of Lebesgue area [S2, S3] for surfaces in normed vector spaces and metric spaces. Then we will show that isometric surfaces in a suitable space $C$ of continuous functions have equal areas from which it will follow that the area defined here for surfaces is a generalization of Lebesgue area defined on surfaces in Euclidean space. It was known that the two areas agreed on surfaces in Euclidean space for which the lower area coincided with the Lebesgue area. To prove that Kolmogorov's Principle holds in $C$ we will require a theorem of the Kirzbraun type.

Let $Q$ be an $n$-cell, $V$ be a normed vector space and $C(Q, V)$ be the space of continuous functions on $Q$ into $V$. If $z \in C(Q, V)$ and if there exists a subdivision of $Q$ into a finite number of $n$-simplexes on each of which $z$ is linear, then $z$ is 
quasilinear. The set of such functions is dense in $C(Q, V)$. If $z$ is quasilinear then we define the elementary area of $z, \mathscr{E}(z)$, by $\mathscr{E}(z)=\sum|z(\Delta)|$ where the summation is taken over the simplexes on which $z$ is linear and, if $z$ is linear on $\Delta$, then $|z(\Delta)|$ $=|\bigwedge d z|$ vol $\Delta$. If $x \in C(Q, V)$ let $L_{V} x=\liminf _{z \rightarrow x} \mathscr{E}(z)$. It is clear that $L_{V} x \leqq L_{W} x$ if $W$ is a vector subspace of $V$ and that there exists a separable subspace $S$ of $V$ such that $L_{S} x=L_{V} x$. Since $S$ can be mapped linearly and isometrically into $m$, the space of bounded sequences, and since isometric surfaces in $m$ have the same area, it follows that $L_{V} x \geqq L_{m} x$ if $x \in C(Q, m)$ and if $X$ and $x$ are isometric. Similarly, if $x$ is continuous on $Q$ into a metric space $\mathscr{M}$ we can define $L_{\mathscr{M}} X=L_{m} x$ for $X$ isometric to $x$. We write $L x$ for $L_{m} x$. If $C$ is a suitable space of continuous functions, then each separable metric space can be mapped isometrically into $C$. After we show that isometric surfaces in $C$ have the same area then, by the above considerations, we will have $L_{C} x=L_{m} y$ whenever $x$ and $y$ are isometric. Thus, when we show that $L_{E_{n}} X$ $=L_{C\left(S^{n-1}\right)} x$ whenever $X$ and $x$ are isometric, we shall be able to conclude that $L$ extends $L_{E_{n}}$.

Lemma 13. Let $(A, \alpha)$ and $(B, \beta)$ be metric spaces and suppose that $A$ is totally bounded. If $x$ is continuous on $A$ into $C(B)$ then $\{x(a) \mid a \in A\}$ is equicontinuous.

Proof. Choose $\varepsilon>0$. There exists $\eta>0$ such that $\left|x(a)-x\left(a^{\prime}\right)\right|<\varepsilon$ whenever $\alpha\left(a, a^{\prime}\right)<\eta$. Since $A$ is totally bounded there exist $a_{1}, \ldots, a_{n} \in A$ such that

$$
A \subset \bigcup_{i=1}^{n}\left\{a \in A \mid \alpha\left(a, a_{i}\right)<\eta\right\}
$$

For each $i=1, \ldots, n$ there exists $\delta_{i}>0$ such that $\left|\left[x\left(a_{i}\right)\right](b)-\left[x\left(a_{i}\right)\right]\left(b^{\prime}\right)\right|<\varepsilon$ whenever $\beta\left(b, b^{\prime}\right)<\delta_{i}$. Let $\delta=\min \delta_{i}$. If $a \in A$ then there exists $j \in\{1, \ldots, n\}$ such that $\alpha\left(a, a_{j}\right)<\eta$. Hence $\left|x(a)-x\left(a_{j}\right)\right|<\varepsilon$. Now if $b, b^{\prime} \in B$ and $\beta\left(b, b^{\prime}\right)<\delta$, we have

$$
\begin{aligned}
\left|[x(a)](b)-[x(a)]\left(b^{\prime}\right)\right| \leqq & \left|[x(a)](b)-\left[x\left(a_{j}\right)\right](b)\right|+\left|\left[x\left(a_{j}\right)\right](b)-\left[x\left(a_{j}\right)\right]\left(b^{\prime}\right)\right| \\
& +\left|\left[x\left(a_{j}\right)\right]\left(b^{\prime}\right)-[x(a)]\left(b^{\prime}\right)\right|<3 \varepsilon .
\end{aligned}
$$

Next we prove a Kirzbraun-type theorem.

THEOREM 5. Let $K$ and $L$ be subsets of $C(B)$ and suppose that $L$ is equicontinuous. If $t: K \rightarrow L$ and if $|t u-t v| \leqq k|u-v|$ for some $k>0$ then there exists $T: C(B) \rightarrow C(B)$ such that $T \mid K=t$ and $|T x-T y| \leqq k|x-y|$.

Proof. If $b \in B, u \in K$ and $v \in C(B)$ let $\psi_{b}(u, v)=t u(b)+k|u-v|$ and let $T v(b)$ $=\inf _{u \in K} \psi_{b}(u, v)$. If $v \in K$ then $\psi_{b}(u, v)-\psi_{b}(v, v)=t u(b)+k|u-v|-t v(b) \geqq k|u-v|$ $-|t u-t v| \geqq 0$ so $T$ extends $t$. Suppose that $\left|w(b)-w\left(b^{\prime}\right)\right|<\varepsilon$ for all $w \in L$ whenever $\beta\left(b, b^{\prime}\right)<\delta$. Then, if $v \in C(B)$,

$$
\begin{aligned}
T v(b)-T v\left(b^{\prime}\right) & =\inf _{u \in K} \psi_{b}(u, v)-\inf _{u \in K} \psi_{b^{\prime}}(u, v) \leqq \sup _{u \in K}\left[\psi_{b}(u, v)-\psi_{b^{\prime}}(u, v)\right] \\
& =\sup _{u \in K}\left[t u(b)-t u\left(b^{\prime}\right)\right] \leqq \varepsilon .
\end{aligned}
$$


Similarly, $T v\left(b^{\prime}\right)-T v(b) \leqq \varepsilon$, so that $T v \in C(B)$. Finally, if $v, w \in C(B)$, then

$$
\begin{aligned}
|T v-T w| & =\sup _{b}|T v(b)-T w(b)|=\sup _{b}\left|\inf _{b} \psi_{b}(u, v)-\inf \psi_{b}(u, w)\right| \\
& \leqq \sup _{b} \sup _{u}\left|\psi_{b}(u, v)-\psi_{b}(u, w)\right|=\sup _{b} \sup _{u}|k| u-v|-k| u-w|| \\
& \leqq k|u-w| .
\end{aligned}
$$

We saw in [S4] that Kirzbraun's Theorem could be used to prove Kolmogorov's Principle. The present version of Kirzbraun's Theorem is all that was actually used in the proof so that we have Kolmogorov's Principle: Let $x$ and $y$ be continuous from an $n$-cell into $C(B)$. Suppose that $\left|x(q)-x\left(q^{\prime}\right)\right| \leqq k\left|y(q)-y\left(q^{\prime}\right)\right|$ for all $q, q^{\prime} \in Q$. Then $L_{C(B)} x \leqq k^{n} L_{C(B)} y$.

Lemma 14. Let $P f=\sum_{i=1}^{n}\left(f, \sigma^{i}\right) \sigma^{i}$ for all $f \in C\left(S^{n-1}\right)$. Then $|P| \leqq \sqrt{ } n$.

Proof. If $f \in C\left(S^{n-1}\right)$ then

$$
\begin{aligned}
|P f|^{2} & =\sum\left(f, \sigma^{i}\right)^{2}=\sum\left\{\frac{1}{K_{n-1}} \int_{S^{n-1}} f \sigma^{i} d \lambda\right\}^{2} \\
& \leqq \sum|f|^{2}=n|f|^{2}
\end{aligned}
$$

THEOREM 6. If $x$ is continuous on $Q$ into Euclidean $n$-space $E^{n}$, then $L_{E^{n}} x=L x$.

Proof. We can identify $E^{n}$ with $E_{n} \subset C\left(S^{n-1}\right)$. Let $z_{k}$ be a sequence of quasilinear functions converging to $x$ in $C\left(Q, C\left(S^{n-1}\right)\right)$ such that $\mathscr{E}\left(z_{k}\right) \rightarrow L x=L_{C\left(S^{n-1}\right)} x$. Then $\left|P z_{k}-x\right|=\left|P z_{k}-P x\right| \leqq|P|\left|z_{k}-x\right| \leqq\left|z_{k}-x\right| \sqrt{ } n$ so that $P x_{k} \rightarrow x$. By Theorem $4 \mathscr{E}\left(P z_{k}\right) \leqq \mathscr{E}\left(z_{k}\right)$. Thus $\cdot L_{E_{n}} x \leqq \lim \inf \mathscr{E}\left(P z_{k}\right) \leqq \lim$ inf $\mathscr{E}\left(z_{k}\right)=L x$. Since the inequality in the other direction is trivial, the theorem is proved.

\section{REFERENCES}

F. W. Fleming, Functions of several variables, Addison-Wesley, Cambridge, Mass., 1965.

S1. E. Silverman, A problem of least area, Pacific J. Math. 14 (1964), 309-331.

S2. - _ Definitions of Lebesgue area for surfaces in metric spaces, Riv. Mat. Univ. Parma 2 (1951), 47-76.

S3. - - Geodesics and Lebesgue area, Proc. Amer. Math. Soc. 15 (1964), 775-780.

S4. - Kirzbraun's Theorem and Kolmogorov's Principle, Proc. Amer. Math. Soc. 17 (1966), 166-169.

Purdue University, LAFAYETTE, INDIANA 